\title{
BMJ Open Clinicians' perspectives of parental decision-making following diagnosis of a severe congenital anomaly: a qualitative study
}

\author{
Robyn Lotto, ${ }^{1}$ Lucy K Smith, ${ }^{2}$ Natalie Armstrong ${ }^{2}$
}

To cite: Lotto R, Smith LK, Armstrong N. Clinicians' perspectives of parental decision-making following diagnosis of a severe congenital anomaly: a qualitative study. BMJ Open 2017;7:e014716. doi:10.1136/ bmjopen-2016-014716

- Prepublication history and additional material are available. To view these files please visit the journal online (http://dx.doi.org/ 10.1136/ bmjopen-2016-014716).

Received 12 0ctober 2016 Revised 29 March 2017 Accepted 3 April 2017

CrossMark

${ }^{1}$ School of Nursing and Allied Health, Liverpool John Moores University, Liverpool, UK

${ }^{2}$ Department of Health Sciences, University of Leicester, Leicester, UK

Correspondence to

Robyn Lotto; r.r.lotto@ljmu.ac.uk

\section{ABSTRACT}

Objective To explore clinicians' perspectives on supporting parents' decision-making following diagnosis of a severe congenital anomaly, and how this is shaped by current policy.

Methods This paper reports data collated as part of a larger project examining parents' decision-making following antenatal diagnosis. The focus of this paper is the data arising from semistructured interviews conducted with 18 clinicians, with findings further supported by data generated from consultations between clinicians and parents. All interviews and consultations were audiorecorded and transcribed verbatim, with analysis based on the constant comparative approach.

Results Three key themes emerged which together shape the practice of clinicians working in this area: first, the law governing termination of pregnancy (TOP) and how clinicians believe this influences the context in which decisions about whether to terminate or continue an affected pregnancy are made; second, approaches to the management of cases seen as particularly challenging; and third, how clinicians understand their role when working with parents. These themes combine to create a strong desire on the part of clinicians for parents to engage in a particular 'rational' form of decision-making and to be able to demonstrate the enactment of this. This is seen as important in order to ensure the 'right' decision has been reached and, particularly when the decision is to terminate, will withstand possible scrutiny.

Conclusions The policy context in which these decisions are made strongly shapes how clinicians practise and what they want to see from the parents with whom they work. The ways in which they seek to overcome the difficulties in interpreting the law may result in variations in the offer of late TOP, both between and within units. This may inadvertently affect the options available to women least able to engage in this idealised form of decisionmaking.

\section{INTRODUCTION}

Around $2 \%-3 \%$ of pregnancies in high-income countries are affected by a congenital anomaly. ${ }^{1}$ In common with many other countries, the UK offers screening that gives the potential for antenatal detection and diagnosis of some of these anomalies. This is

\section{Strengths and limitations of this study}

- This qualitative study provided an in-depth exploration of how policy context both shapes clinicians' practice and their expectations of the parents with whom they work.

> Combining semistructured interviews and consultation recordings enabled us to both identify the difficulties clinicians encounter and hear examples of these in practice.

- The heterogeneous nature of the clinician sample reflects the multidisciplinary/specialty counselling style adopted in practice, thus strengthening the validity of the findings presented.

- The sites from which the sample was drawn were all specialist referral centres. The transferability of these findings to smaller district general hospitals should not be assumed.

offered via the NHS Fetal Anomaly Screening Programme (FASP), which provides screening for major anomalies where it is indicated that 'the baby may die shortly after birth; there may be a benefit from treatment before birth; to facilitate planned delivery in an appropriate hospital/centre; and/or to optimise treatment after the baby is born and have detection rates which exceed $50 \%{ }^{\prime}{ }^{2}$ Targets are set for the antenatal identification of 11 selected anomalies. These are serious cardiac, anencephaly, spina bifida, exomphalos, renal agenesis, lethal skeletal dysplasia, congenital diaphragmatic hernia, trisomies 13 and 18, cleft lip and gastroschisis. ${ }^{3}$ Where mortality related to the condition is deemed high, as is the case for the first nine anomalies listed (FASP9), diagnosis may result in the offer of a termination of pregnancy (TOP). In England and Wales, around $70 \%$ of such pregnancies result in a termination. ${ }^{4}$ Nonetheless, congenital anomalies account for around 30\% of neonatal and infant mortality across the UK. ${ }^{5}$

The 1990 Human Fertilisation and Embryology Bill provided the latest amendment to 
the 1967 Abortion Act. In England and Wales, TOP for a severe congenital anomaly is performed under Clause $\mathrm{E}$ of the Act, where no gestational limit exists. This is in contrast to a TOP undertaken to protect a woman's health, which is performed under Clause $\mathrm{C}$ and only permitted up to 24 weeks' completed gestation. These abortions are frequently referred to as an abortion for 'social reasons'. ${ }^{6}$ To be eligible for TOP under Clause E, the anomaly must be classified as severe where there is a substantial risk for grave permanent injury or serious disability. ${ }^{7}$ The number of late terminations, namely those undertaken under Clause E after 24 weeks' gestation, are relatively few, with 230 such terminations recorded by the Department of Health in 2015 across England and Wales.

Interpretation of the terms substantial and severe is essentially a clinical decision and responsibility. Where the requirements of the law are perceived as having been met, the subsequent decision to continue or terminate the affected pregnancy is made in partnership between clinicians and parents, and requires agreement of at least two doctors. Professional and legal guidance exists to support 'good practice' in respect to these interactions. In particular, the broader issues pertaining to non-directive counselling and informed, shared decision-making feature prominently in the literature, with the tensions and difficulties encountered by clinicians well documented. ${ }^{9-13}$ Defining the boundaries between choice and coercion by clinicians working in this field, and the subsequent ethical issues arising from these discussions, highlights the complexity of the field within which clinicians practise. However, little is known about the experiences and views of clinicians as they navigate the law and facilitate the decision-making process within this context. Existing evidence suggests a 'hardening' in clinicians' assessments of what constitutes a severe anomaly, with the requirements in terms of prognosis becoming more stringent. ${ }^{14}$ However, a recent study examining professional views of TOP for non-lethal anomalies highlighted that despite the contentiousness of this debate within the public domain, the clinicians involved were able to negotiate a moral pathway through the issues. ${ }^{15}$

This paper seeks to provide insight into clinicians' perspectives on both supporting parents' decision-making following diagnosis of a severe congenital anomaly, and how this is shaped by current policy. This will enable us to better determine policy to support clinicians as they care for parents. While the contextual framework in which these clinicians practise is determined by UK law and policies, the findings presented within this study go beyond the confines of a single legal framework.

\section{METHODS}

This paper reports data collected as part of a larger project examining parents' decision-making following antenatal diagnosis of an FASP9 anomaly. A qualitative approach comprising one-to-one interviews with clinicians and parents and recordings of consultations between them was employed in order to best understand the complex reality of such decision-making. ${ }^{16}$ This produced detailed, contextualised descriptions of behaviour and accounts of how people made sense of the situations in which they found themselves.

Here the principal focus is data from the clinician interviews. A total of 22 clinicians were invited to participate from across four tertiary referral centres (across two hospital trusts). Sampling was purposive to represent a range of healthcare professionals, including fetal medicine consultants and midwives, specialist fetal cardiology and neurology consultants, neonatologists and others working within the fetal medicine specialty. Of the 22 approached, one declined due to imminent retirement, one withdrew due to time constraints, and two interviews were started but interrupted by clinical commitments and not ultimately used at participants' request. The final sample of 18 participants was heterogeneous in terms of gender, with a 50/50 split; age, ranging from early 30 s to late $60 \mathrm{~s}$; and length of time working within the fetal medicine setting, spanning from 3 to $20+$ years. All fetal medicine and specialty fetal consultants employed across the four sites were interviewed and data were included in the study. Additional participants from supporting specialities were identified and selected for recruitment based on active involvement in antenatal counselling following suspicion or diagnosis of a severe congenital anomaly. Due to the sensitivity of the subject area, no further breakdown of the sample is given in order to protect the anonymity of those involved. The sample size was not predetermined. Similar themes arose within all the interviews and the analytical themes were not significantly modified after the first 10 interviews. However, in order to ensure recruitment of clinicians working within a range of supporting specialities, we continued recruitment after theoretical saturation had been achieved. ${ }^{17}$ Opportunities for participant checking of findings were provided throughout the analysis process.

Participant information was provided and written consent obtained. Semistructured interviews were digitally recorded, anonymised and transcribed verbatim. Participants were invited to review their transcripts. Topics covered included perspectives on TOP; national and local policies governing TOP, in particular late termination; interpretation of the law in these cases; and views on parental decision-making post diagnosis (see online supplementary file). Interviews lasted on average 1 hour, ranging between $50 \mathrm{~min}$ and 1 hour $40 \mathrm{~min}$. All interviews were undertaken by RL. None of the researchers worked in the clinical area or had prior knowledge of the participants. Parents-to-be were recruited in the fetal medicine clinic. Following formal documentation of their consent, consultations were audio-recorded and transcribed verbatim.

Data analysis used a constant comparative-based approach, ${ }^{18}$ with NVivo software used to assist organisation. An inductive approach was adopted and analysis undertaken concurrently with the ongoing data 
collection, thus enabling the further exploration of emergent themes. An amended one sheet of paper method was employed alongside to assist with visualisation of the data. ${ }^{19}$ This involved coding extracts, creating concepts and connections and finally merging them into the themes presented. Data generated from consultations provided the opportunity to contextualise and explore the ways in which clinicians talked about and sought to overcome the difficulties they perceive.

A reflective diary, completed immediately after each interview, provided additional context. Four randomly selected interviews were coded separately by the research team and compared across the team. Consensus on emergent themes was reached through regular discussions. Illustrative quotes are presented in the findings.

Ethical permission was granted by the Nottingham Research Ethics Committee (REC Reference 13/ $\mathrm{EM} / 0293)$.

\section{FINDINGS}

While the focus of the study was 'decision-making' following suspicion or diagnosis of a severe congenital anomaly, heavy emphasis was placed on TOP by the clinicians. Three key themes emerged which together shape the practice of clinicians working in this area: the influence of the law governing TOP, approaches to management of challenging cases and clinicians' understanding of their role when working with parents. These themes combine to create a strong desire on the part of clinicians for parents to engage and enact in a particular 'rational' form of decision-making. This is seen as important in order to ensure the 'right' decision has been reached and, particularly when the decision is to terminate, would withstand possible scrutiny, in particular legal challenges.

\section{Influence of the law governing TOP}

Although the guidance is intended to inform practice, clinicians were troubled by the degree to which the terminology used is subjective and open to interpretation. The difficulty in defining and gaining consensus for a shared understanding of what constitutes a severe congenital anomaly, and what does not, was widely discussed.

"You can't! ... so when we debated at the British Maternal and Fetal Medicine Society, whether [an anomaly] met the criteria for Clause E, severe, the audience was completely divided ... So we can't agree on that." (Clinician 09)

For many clinicians, the law governing TOP was regarded as having been intentionally written in a 'woolly' manner, and symptomatic of a failure of society as a whole to really address this sensitive and complex issue.

"I mean the termination laws ... aren't well thought through and society has just allowed this to continue because it's a problem they don't want to address." (Clinician 08)

They expressed frustration over this, suggesting that the emotiveness of the issue had resulted in a failure of society to discuss openly and reach a consensus on where the boundary of severity should lie. Instead, the responsibility was, they felt, being laid on individual clinicians who were then subjected to intense scrutiny. This left many clinicians feeling vulnerable.

"I have an ongoing degree of anxiety ... that what we're doing is legal but there's a huge tranche of the population who would say ... 'it's wrong what they are doing.' And... mostly [from] people who haven't had to make these choices." (Clinician 04)

In some cases, fear of scrutiny appeared to restrict the options clinicians felt able to make available to parents, risking time constraints being applied to the decision-making, so that TOP could be performed before 24 weeks' gestation.

"I would ... encourage people to make a choice ... before 24 weeks' gestation because I don't think either myself professionally, personally or for them as individuals we particularly want anybody to be more carefully scrutinised as to why they've chosen a termination beyond [that gestation]. Nobody will look a second time if it is less than 24 weeks."

(Clinician 10)

After 24 weeks' gestation, individual clinicians' thresholds for offering a TOP appeared to become more stringent. When discussing the possibility of offering a TOP for ventriculomegaly, one clinician commented:

"Before 24 weeks a termination could be offered for a ventriculomegaly of $14 \mathrm{~mm}$ for instance, but after 24 weeks that wouldn't necessarily fulfil the definition [of severe]." (Clinician 13)

The change in method by which data for TOPs performed beyond 24 weeks is collected, whereby clinicians are responsible for submitting additional documentation to the Department of Health, created an added sense of unease.

"There is a sense that the data are submitted into a black hole and it's unclear who gets to see this." (Clinician 04)

\section{Approaches to management of challenging cases}

In order to protect themselves while working in what all acknowledged to be an unclear and difficult context, clinicians talked about approaches to the management of cases they found particularly challenging. One important strategy involved overcoming these pressures and standardising practice within a centre through the use of a 'corporate' approach to interpreting the law. In practice, this involved regular multidisciplinary meetings and formal peer support processes. This served to remove the responsibility from an individual by placing it on a group, but also theoretically overcame some of the differences between clinicians' practice, as it allowed for discussion.

“... I have my own spectrum, my own gradation and certainty. In ...cases where it's not obvious, we sit together... 
and listen to one another's opinions. That has altered the initial thoughts ...about whether we would offer a late termination." (Clinician 10)

Although mechanisms such as this appeared to provide reassurance to individual clinicians and help reduce variation between clinicians within a centre, they are unlikely to reduce intercentre variation. Instead, some centres elsewhere were referred to as being 'more termination minded' than others, highlighting the differing interpretations of the law. Clinicians talked about referring parents to these other centres for second opinions when a TOP beyond 24 weeks' gestation was requested, but they were not willing to perform one. As above, the impact of this gestational cut-off was significant, beyond this clinicians became more stringent, deliberately avoiding possible scrutiny.

“... before 24 weeks ... I could've organised that ... but after 24 weeks... I couldn't, even though [the parents] felt quite strongly they wanted it ... it was my obligation to refer them on to somebody else [who would offer a termination]." (Clinician 03)

A further mechanism identified was referral to a clinical ethics committee. These committees consist of a number of clinical and ethical/legal advisors who discuss individual cases, with referrals increasing significantly over the past decade. ${ }^{20} \mathrm{~A}$ number of the clinicians identified the importance of the ethics committee opinion, particularly in cases of late TOP where consensus had not been reached with the parents.

"It was actually really useful to have the ethics opinion so it was as if we had some back up from a third party, from an independent third party." (Clinician 02)

Although the committee provides guidance rather than a legally binding decision, referral to the committee was perceived as a protective mechanism in the event of future litigation, with clinicians wary of acting against the findings of the committee.

“... no-one locally would have been supportive as ethics had said no. So she would have had to go somewhere else to explore other views..." (Clinician 04)

\section{Clinicians' understanding of their role when working with parents}

Across the interviews, the clinicians expressed a shared understanding of their professional roles and responsibilities. They saw their over-riding function to be that of facilitator in assisting parents to make the right decision for them. In order to achieve this, they discussed adopting a number of roles throughout the decision-making process: first, was that of information bearer and interpreter; second, assessor of the decision-making process; and finally, supporter of the final decision, whatever it might be.
Clinicians emphasised the importance of their role as an information bearer, stressing the need for the information to be given impartially, in a non-directive manner.

“... the objective of antenatal counselling is to give them all the facts so they can make a decision. But we never ever try to influence them one way or the other." (Clinician 14)

A clear distinction was made by the clinicians between the decision-making process and the decision itself, where the 'right' decision could be achieved by engaging actively in the decision-making process.

“There isn't a right or wrong decision... it's making sure you have all the information, and weigh it up so that you make the right decision for you and your family at that time."

(Clinician 11)

Their second role involved assessing the decision-making process; here clinicians sought evidence that the parents had actively engaged and had been transparent in their deliberation over the best course of action for them.

"[I have] a duty to explore how she [the mother] has reached her decision." (Clinician 16)

Finally, the importance of their role as a supporter of the decision, whatever it might be, was universally endorsed by the clinicians interviewed.

"Because it's a huge decision to terminate your baby and if they've chosen to, then it's important that you make them feel as good as possible about their decision. And if they've chosen to carry on then it's important to talk about the good side of that..." (Clinician 18)

\section{Desire for 'rational' decision-making}

The three themes outlined above combined to create a strong desire on the part of clinicians for parents to engage in a particular 'rational' form of decision-making and to be able to demonstrate the enactment of this. Theoretically, rationality has been defined as 'a style of behaviour that is appropriate to the achievement of given goals, within the limits imposed by given conditions and constraints. ${ }^{21}$ From the perspective of the clinicians, requirements of this 'rational' process involved parents actively engaging, weighing up relevant information, deliberating over the future impact of the decision, balancing risk in terms of likelihood as well as severity of predicted disability, and finally implementing the decision. While clinicians shared a well-defined understanding of their own role in the decision-making process, they also had expectations of and placed responsibilities on parents. Clinicians wanted to see parents engage in a systematic, 'rational' approach to the decision-making process in order to be confident in any decision made.

“... it's important that they demonstrate a rational decisionmaking process [which will ultimately] result in the right decision." (Clinician 16) 
Where parents did not engage in a 'rational' decision-making process, clinicians expressed concern that parents risked continuing the pregnancy by default. This was typically interpreted as a passive choice, with clinicians expressing concerns over either parental willingness or ability to actively engage in the decision-making process.

“... some people... their default position is to continue with the pregnancy because to actually go through that process of thinking where might I be, what might the consequences be... they're either not willing to do that, or they really just don't have the skills." (Clinician 10)

For clinicians, a 'successful outcome' of the decision-making process was one where they were satisfied that a rational decision-making process had been employed.

"If we reach an agreement that she's satisfied with the information, and I am satisfied with the decision she's made, there's a successful relationship and a successful outcome." (Clinician 09)

The importance of the process is clarified further into the interview.

\section{"[The important thing] is to explore how she has reached her decision." (Clinician 09)}

As a consequence, tensions could arise when clinicians did not believe that parents had fulfilled their role. Where parents requested a TOP but clinicians were not satisfied with how they had reached this decision, clinicians talked about how they might put barriers in place to ensure that the expected processes were undertaken by parents prior to any procedure being sanctioned.

... “... 'cause ultimately I don't have to offer a termination. I have to offer them the opportunity of a second opinion elsewhere... but mostly if I say that I'm not 100\% comfortable with this, at the very least I want you to listen to what someone else has to say." (Clinician 07)

On occasions, these actions resulted in a breakdown of the parent-clinician relationship, with subsequent counselling becoming defensive.

\section{"After they had gone for the second opinion they phoned back again and asked why the amniocentesis hadn't been repeated. It was explained that there was no point in repeating the test. [The consultant providing the second opinion] said they are a very difficult couple and we need to be very clear in what we are saying to them." (Clinician 09)}

\section{DISCUSSION}

This paper has explored clinicians' perspectives on the legal and policy context governing TOP and the decision-making processes following suspicion or diagnosis of a severe congenital anomaly. While the interviews were framed primarily to explore perceptions of decision-making, the data generated by the clinicians focused heavily on TOP and its implications. This became particularly pertinent after 24 weeks, the point at which a TOP is no longer permitted under Clause $\mathrm{C}$ and can only be undertaken under Clause $\mathrm{E}$ for reasons of a severe anomaly. Clinicians found the law around TOP in these circumstances difficult to interpret, and 'woolly'. In particular, difficulties were highlighted in identifying the point at which a severe anomaly might become incompatible with a reasonable quality of life, resulting in the need for personal and professional judgements to be made. For clinicians, managing these dilemmas alongside ever-increasing public scrutiny is challenging.

The way in which the law is interpreted is open to legal challenge, and criminal charges, with the issues created by the relatively recent Jepson case, in which a legal challenge to the late abortion of a fetus with cleft lip and palate was made, still resounding in many clinicians' ears. ${ }^{22}$ Although the case was finally dismissed in 2005, it has been suggested that ensuing scrutiny resulted in making an already distressing situation for expectant parents and their doctors even more difficult. ${ }^{23}$ While previous studies have highlighted the complexity of their role, and the difficulties encountered, ${ }^{24}$ the findings of this study highlight the mechanisms employed by clinicians in navigating these complexities. One predominant mechanism was the 'corporate approach' to clinical decision-making.

While decision-making is commonly enacted through multidisciplinary meetings, variations between individuals and units in relation to practice in this field were widely acknowledged, with parents being referred to more 'termination-minded' colleagues or units when consensus could not be reached between clinicians themselves or between clinician and parent. These informal 'second opinion'pathways appeared well embedded in practice. One of the risks associated with having a corporate perspective on TOP is that it creates variations in access to treatment. Although the option for a second opinion was theoretically available for all women, in practice the cost emotionally, financially and in terms of time may act as an important barrier to access for some parents.

Since the inception of the UK Clinical Ethics Network in $2001,{ }^{25}$ there has also been a gradual increase in referral of cases to ethics committees. ${ }^{20}{ }^{26}$ However, the subjective nature of the decision means that consistency of opinion is not always guaranteed between committees. ${ }^{27}$

Where the offer of termination under Clause $\mathrm{E}$ is reliant on individual or corporate interpretation of the law, the creation of geographical variation in the offer of terminations is inevitable. Formal and informal processes such as referral to a clinical ethics committee or 'more termination minded' colleagues or units are designed to reduce these variations. However, the increasing referral rate of complex cases to a clinical ethics committee is perhaps more reflective of an increasingly litigious society, where the clinical ethics committee serves to protect the clinician, particularly in scenarios where a lack of consensus between parent and clinician exists. For parents seeking treatment in units further from home, significant costs 
(travel and time) are incurred. In addition, a level of health literacy in order to engage with clinicians and to instigate discussions is required. ${ }^{28}$ These barriers are likely to have a particularly significant impact on parentsto-be from areas of high deprivation and ethnic minority groups, both in terms of the financial implications of accessing a second opinion and in relation to their ability to engage in discussions leading to referral. While lower rates of TOP among women living in more deprived areas compared with those living in less deprived areas ${ }^{29}$ may be a result of parental choice, possible systematic variations in access to or provision of services, and the role clinicians play as gatekeepers, the impact of such issues becomes particularly pertinent.

One solution broached has been the clarification of the law and construction of a list of conditions that meet the severity criteria for Clause E. ${ }^{30}$ However, this proposal has the potential to restrict the options open to parents, through failing to encapsulate the variability observed within a single diagnosis, as well as the impact of the coexistence of other anomalies, ${ }^{24}$ and fails to account for the difficulties in reaching a consensus for the definition of a severe condition. It is clear there is no simple solution. Social attitudes to TOP will continue to impact on clinicians' interpretation of the law, with mechanisms to separate the two warranting exploration. Moves to decriminalise abortion, whereby the decision to terminate will be judged as a medical rather than legal matter, are currently under debate. ${ }^{31}$ While the likelihood of this proposal becoming law is unclear, figures from Canada, where abortion was decriminalised over 30 years ago, suggest that little will change in terms of the number of abortions performed. ${ }^{32}$ However, the impact of decriminalisation on social attitudes warrants further study. Clinicians perceived their over-riding role within the decision-making process to be that of facilitator. Essential characteristics of the role included those of information bearer and interpreter, assessor of the decision-making process and finally supporter of the decision.

The clinicians made a clear demarcation between the decision-making process and the decision itself, where the prerequisite of a 'right' decision was active engagement by parents in the decision-making process. Failure of parents to engage in this manner generated high levels of concern. This was particularly evident when parents continued the affected pregnancy, by 'default', where no active decision appeared to have been made. Much of the literature relating to decision-making theories focuses on rationality and the coherence of the decision-making process. ${ }^{33}$ This is clearly reflected in the responses required by clinicians. Where parents did not adhere to these expectations, clinicians reported high levels of stress and discomfort. In these instances, the parent-clinician relationship risked deteriorating, risking disengagement by parents from the process, and thus continuation of a pregnancy by 'default'. While it is likely that the decision-making processes of some parents reflect the desired attributes highlighted by clinicians, others will not. A synergy between the actions of parents and expectations of clinicians is likely to facilitate the decision-making process. Conversely, conflict between actions and expectations risks the creation of barriers. While little is known about the decision-making processes of women and their partners, the impact of clinicians' expectations is likely to intensify variations in the way the decision-making process is enacted.

We acknowledge this study has limitations. First, it is based on a relatively small sample of clinicians, although the professional groups represented are diverse. Second, the four units from where participants were recruited represent a small number of the fetal medicine units across England and Wales. The importance of the corporate context in which clinicians practise was highlighted, and may therefore limit the transferability of some of the findings to other settings, in particular smaller, non-specialist units. Despite these limitations, these data represent an important contribution to the knowledge and understanding of the context in which decision-making following diagnosis of a severe congenital anomaly is made and how clinicians understand their role within this. While this study provides a preliminary insight into the decision-making process following diagnosis of a severe congenital anomaly, from the perspective of the clinicians, in order to identify how the variations in the decision to continue or terminate an affected pregnancy across levels of deprivation arise and subsequently address the observed inequalities in neonatal and infant mortality, a better understanding of the decision-making process itself is required. While there has been a recent increase in interest in aspects of parental decision-making, either from the perspective of those who terminated an affected pregnancy ${ }^{34-36}$ or those who continued, ${ }^{37-39}$ little literature reflects the processes undertaken to get to that point.

Due to the emotive nature of TOP, public scrutiny is unlikely to reduce. Increasingly, processes have been incorporated into clinical practice to distance individual clinicians from this. However, these processes may inadvertently result in variations in the care parents receive. While for some parents, these will facilitate the decision-making process, for others they will act as barriers. This remains a complex problem with no simple solution. Clarification of the law is unlikely to be welcomed by clinicians, as the complexity and variability of the conditions encountered make listing of conditions impractical. From a legal perspective, further discussion is required around the risk of criminalisation of clinicians who perform late TOP.

Contributors RL, LKS and NA conceived and designed the study. RL was responsible for acquisition of data. All authors contributed to interpretation of data. RL wrote the first draft of the paper. All authors were involved in subsequent revision and approved the final manuscript. $\mathrm{RL}$ is the guarantor.

Funding This study was funded by a University of Leicester Health Sciences Department grant between TIMMS (The Infant Mortality and Morbidity Studies Group) and SAPPHIRE (Social Sciences Applied to Healthcare Improvement Research). LKS is funded by a National Institute for Health Research Career 
Development Fellowship. This article presents independent research funded by the National Institute for Health Research (NIHR). The views expressed are those of the authors and not necessarily those of the NHS, the NIHR or the Department of Health.

\section{Competing interests None declared.}

Ethics approval Nottingham Research Ethics Committee (REC Reference 13/ $\mathrm{EM} / 0293)$.

Provenance and peer review Not commissioned; externally peer reviewed.

Data sharing statement № additional data are available from the authors.

Open Access This is an Open Access article distributed in accordance with the Creative Commons Attribution Non Commercial (CC BY-NC 4.0) license, which permits others to distribute, remix, adapt, build upon this work non-commercially, and license their derivative works on different terms, provided the original work is properly cited and the use is non-commercial. See: http://creativecommons.org/ licenses/by-nc/4.0/

(C) Article author(s) (or their employer(s) unless otherwise stated in the text of the article) 2017. All rights reserved. No commercial use is permitted unless otherwise expressly granted.

\section{REFERENCES}

1. Askelsdóttir B, Conroy S, Rempel G. From diagnosis to birth: parents' experience when expecting a child with congenital anomaly. Adv Neonatal Care 2008;8:348-54.

2. Fetal anomaly screening programme - remit, aims and objectives (Internet). 2009 http://fetalanomaly.screening.nhs.uk/ fetalanomalyresource/images/stories/Downloads/2.1/FASP-RemitAims-Objectives-May09.pdf (accessed 09 May 2015).

3. Fetal anomaly screening standards (Internet). $2010 \mathrm{http}: / /$ fetalanomaly.screening.nhs.uk/standards (accessed 10/2015).

4. Department of Health. Abortion statistics in England and Wales (Internet). 2015 https://www.gov.uk/government/uploads/system/ uploads/attachment_data/file/570040/Updated_Abortion_Statistics_ 2015.pdf (accessed 06/2016).

5. Budd JL, Draper ES, Lotto RR, et al. Socioeconomic inequalities in pregnancy outcome associated with down syndrome: a populationbased study. Arch Dis Child Fetal Neonatal Ed 2015;100:F400-F404.

6. Oakley L, Maconochie N, Doyle P, et al. Multivariate analysis of infant death in England and Wales in 2005-06, with focus on socioeconomic status and deprivation. Health Stat Q 2009:22-39.

7. Bryant LD, Green JM, Hewison J. Prenatal screening for down's syndrome: some psychosocial implications of a 'screening for all' policy. Public Health 2001;115:356-8.

8. Human fertilisationand embryology act (Internet). $1990 \mathrm{http}: / / \mathrm{www}$ legislation.gov.uk/ukpga/1990/37/contents (accessed 10/2015)

9. Aite L, Zaccara A, Mirante N, et al. Antenatal diagnosis of congenital anomaly: a really traumatic experience? J Perinatol 2011;31:760-3.

10. Williams $C$, Alderson $P$, Farsides $B$. Is nondirectiveness possible within the context of antenatal screening and testing? Soc Sci Med 2002;54:339-47.

11. Press $\mathrm{N}$, Browner $\mathrm{CH}$. Why women say yes to prenatal diagnosis. Soc Sci Med 1997;45:979-89.

12. Al-Jader LN, Parry-Langdon N, Smith RJ. Survey of attitudes of pregnant women towards down syndrome screening. Prenat Diagn 2000;20:23-9.

13. Heyman B, Hundt G, Sandall J, et al. On being at higher risk: a qualitative study of prenatal screening for chromosomal anomalies. Soc Sci Med 2006;62:2360-72.

14. Statham H, Solomou W, Green JM. Communication of prenatal screening and diagnosis results to primary-care health professionals. Public Health 2003;117:348-57.
15. Crowe L, Graham R, Robson S, et al. Poster 25 - Negotiating acceptable termination of pregnancy for non-lethal fetal anomalies. professional views. Br J Obstet Gynaecol 2015:101.

16. Mason J. Qualitative researching. London: Sage Publications Ltd, 2002.

17. Bryman A. Social Research Methods. 1st ed. New York: Oxford University Press, 2001

18. Glaser BG, Strauss AL. The discovery of grounded theory: strategies for qualitative research. 7th ed. New Jersey: Transaction Publishers, 2012.

19. Ziebland S, McPherson A. Making sense of qualitative data analysis: an introduction with illustrations from DIPEx (personal experiences of health and illness). Med Educ 2006;40:405-14.

20. Slowther AM, McClimans L, Price C. Development of clinical ethics services in the UK: a national survey. J Med Ethics 2012;38:210-4.

21. Simon HA. From Substantive to Procedural Rationality. Latsis SJ, ed. Method and appraisal in Economics. Cambridge: Cambridge University Press, 1976:129-48.

22. Dyer C. Doctors who performed late abortion will not be prosecuted. BMJ 2005;330:688.

23. Britains abortion law (Internet). 2013 http://www.reproductivereview. org/images/uploads/Britains_abortion_law.pdf. (accessed 07/2015).

24. Statham H, Solomou W, Green J. Late termination of pregnancy: law, policy and decision making in four english fetal medicine units:. BJOG 2006;113:1402-11.

25. Slowther A, Johnston C, Goodall J, et al. A practical guide for clinical ethics support. ETHOX: University of Oxford, 2004.

26. McLean SAM. What and who are clinical ethics committees for? $J$ Med Ethics 2007;33:497-500.

27. Angell E, Sutton AJ, Windridge K, et al. Consistency in decision making by research ethics committees: a controlled comparison. $J$ Med Ethics 2006;32:662-4.

28. Beauchamp A, Buchbinder R, Dodson S, et al. Distribution of health literacy strengths and weaknesses across socio-demographic groups: a cross-sectional survey using the Health literacy questionnaire (HLQ). BMC Public Health 2015;15:678-90.

29. Smith LK, Budd JL, Field DJ, et al. Socioeconomic inequalities in outcome of pregnancy and neonatal mortality associated with congenital anomalies: population based study. BMJ 2011;343:d4306.

30. Savulescu J. Is current practice around late termination of pregnancy eugenic and discriminatory? maternal interests and abortion. J Med Ethics 2001;27:165-71.

31. Dyer C. Decriminalisation of abortion. BMJ 2017;356:j1485

32. Norman WV, Downie J. Abortion care in Canada is decided between a woman and her doctor, without recourse to criminal law. BMJ 2017;356:j1506.

33. Bijma $\mathrm{HH}$, Wildschut $\mathrm{HI}$, Van $\mathrm{DH}$, et al. Parental decision-making after ultrasound diagnosis of a serious foetal abnormality. Fetal Diagn Ther 2005;20:321-7.

34. Lotto R, Armstrong N, Smith LK. Care provision during termination of pregnancy following diagnosis of a severe congenital anomaly - A qualitative study of what is important to parents. Midwifery 2016;43:14-20.

35. Carlsson T, Bergman G, Karlsson AM, et al. Experiences of termination of pregnancy for a fetal anomaly: a qualitative study of virtual community messages. Midwifery 2016;41:54-60.

36. Fisher J, Lafarge C. Women's experience of care when undergoing termination of pregnancy for fetal anomaly in England. J Reprod Infant Psychol 2015;33:69-87.

37. McCoyd J. Critical Aspects of Decision-Making and Grieving After Diagnosis of Fetal Anomaly. In: Paley Galst J, Verp MS, eds. Prenatal and Preimplantation diagnosis the burden of Choice Springer International Publishing, 2015:269-85.

38. Côté-Arsenault D, Denney-Koelsch E. "Have no regrets:" Parents experiences and developmental tasks in pregnancy with a lethal fetal diagnosis. Soc Sci Med 2016;154:100-9.

39. Coleman PK. Diagnosis of Fetal Anomaly and the Increased Maternal Psychological Toll Associated with Pregnancy Termination. Issues Law Med 2015;30:3-23. 